\title{
L'intégration de la classe virtuelle synchrone à l'université, un levier de transformation de la professionnalité enseignante?
}

\section{Synchronous online classrooms integration at university, a transformation way in teacher professionality?}

\section{¿La aula virtual sincrónica integración en la universidad, una herramienta para transformar la profesionalidad de los profesores?}

\author{
Béatrice Verquin Savarieau, Maîtresse de conférences \\ Cirnef, Université de Rouen Normandie, France \\ beatrice.savarieau@univ-rouen.fr
}

Hervé Daguet, Maître de conférences

Cirnef, Université de Rouen Normandie, France

herve.daguet@univ-rouen.fr

L'intégration des classes virtuelles synchrones modifie-t-elle la professionnalité enseignante et quelles sont les transformations qui en résultent? Cette recherche réalisée auprès des enseignants du campus numérique Forse de l'université de Rouen illustre que les dispositifs de communication médiatisée rendent possible de nouvelles modalités d'intervention, en soirée et dans la cadre de ce qui pourrait relever du télétravail. C'est donc l'agir professionnel enseignant qui s'en trouve questionné, dans ses dimensions institutionnelles, épistémiques et ce qui fonde l'agir professionnel enseignant dans son expression, comme l'absence du corps instituant de l'enseignant. 
Mots-clés: classe virtuelle synchrone, professionnalité enseignante, intégration technologique, dispositif de communication médiatisée, formation à distance, multimodalité

ABSTRACT

Do synchronous online classrooms modify teacher professionality, and what are the resulting transformations? This research conducted with teachers at Forse digital campus at the University of Rouen illustrates that media-based communication devices make possible new ways of intervening for evening classes and teleworking. It is thus the professional teaching act that is questioned in its institutional, epistemic dimensions and the foundation of the expression of professional teaching action such as the absence of the instituting body of the teacher.

Keywords: virtual classroom synchronous, teacher professionality, technology integration, ICT communication device, transactional distance, multimodality

RESUMEN

¿El uso de aulas virtuales sincrónicas modifica la profesión docente? ¿cuáles son las transformaciones que resultan de ello? Esta investigación realizada con docentes del campus digital Forse de la Universidad de Rouen ilustra que los dispositivos de comunicación mediatizados permiten nuevas formas de intervención, en horario nocturno y en el contexto de lo que podría considerarse teletrabajo. Por lo tanto, lo que se cuestiona es el acto de enseñanza profesional, en sus dimensiones institucionales y epistémicas, así como lo que fundamenta la acción docente profesional como tal, como la ausencia física del docente.

Palabras clave: aula virtual sincrónica, integración tecnológica, profesionalidad de los docentes, dispositivo de comunicación mediado, aprendizaje a distancia, multimodalidad

\section{Introduction}

L'intégration des classes virtuelles synchrones dans l'agir professionnel des enseignants du supérieur estelle source de transformations? En quoi modifie-t-elle la professionnalité enseignante et l'espace d'intervention des enseignants? Cette recherche s'inscrit en enseignement supérieur, dans la cadre du campus numérique Forse de l'université de Rouen. Nous définissons la professionnalité comme étant manifeste d'un ensemble de normes et de valeurs enracinées dans des situations, dans un processus historique et dans un à-venir du métier. Nous l'aborderons ici essentiellement sous l'angle du développement d'apprentissages nouveaux directement en lien avec l'activité professionnelle exercée; tandis que celui de l'expertise construite et partagée par les membres d'un groupe professionnel sera peu développé.

Dans un premier temps, nous présenterons succinctement le campus numérique Forse, afin de pouvoir situer le contexte de cette recherche. Dans un second, nous décrirons l'outil classe virtuelle Adobe "Connect " ${ }^{\mathrm{TM}}$ et les questions qui en résultent, notamment en termes de méthodologie de recherche en technologies éducatives permettant la prise en compte de la multimodalité (communication écrite et orale). 
Enfin nous préciserons quelques résultats, dans la continuité des travaux que nous avons déjà publiés sur les usages des classes virtuelles (Verquin Savarieau et Daguet, 2012; Daguet et Savarieau, 2014; Verquin Savarieau et Daguet 2016), mais davantage du point de vue des effets perçus sur la transformation de la professionnalité enseignante. Nous pouvons souligner que si de nombreux travaux ont été consacrés à la formation à distance du point de vue des étudiants, plus rares sont ceux consacrés à l'expérience vécue par les enseignants.

\section{Les classes virtuelles, une valeur ajoutée pédagogique qui permet d'accompagner les dispositifs distanciels}

La campus numérique "Forse" (Formations et Ressources en Sciences de l'Education) de l'université de Rouen, est né de l'appel à projets dit " campus numérique " (Thibault, 2007) émanant du ministère de l'enseignement supérieur et de la recherche français en 2002. Nous pouvons considérer que la pérennité du campus FORSE témoigne d'une certaine réussite (Deceuninck, 2007; Wallet, 2007) et d'une capacité à mettre en œuvre l'innovation dans les pratiques pédagogiques par l'usage des artefacts numériques. Ainsi comme le rappelait Béziat (2018), ce dispositif de formation qui propose un cursus en Sciences de l'Education (de la licence au master) est porté par un consortium composé les universités de Rouen et de Lyon 2 et par le Centre national d'enseignement à distance (Cned). Si les premières modalités d'accompagnement tutoral prenaient en compte les outils de communication disponible alors (minitel, téléphone, courrier et, pour ceux qui en étaient équipés, messagerie électronique sur connexion internet bas débit), il n'en est plus de même aujourd'hui. La généralisation de la connexion haut débit à conduit l'équipe pédagogique à assurer un enseignement tout en ligne (suppression des cours par correspondance et donc des supports papier), médiatisation des cours sur la plateforme Moodle ${ }^{\mathrm{TM}}$, tutorat par internet, notamment par l'intégration des classes virtuelles depuis 2010. Les scénarios pédagogiques mis en œuvre au sein de ce campus se sont diversifiés, tant pour aller vers des scénarios collaboratifs que pour intégrer les instruments numériques dédiés à la collaboration (classes virtuelles, travaux collaboratifs tutorés) ou du web social (Facebook ${ }^{\mathrm{TM}}$, Second Life $^{\mathrm{TM}}$, etc...).

Nous retenons que les nombreux effectifs en licence (350 en L3) conduisent les ambitions pédagogiques à se focaliser sur la préparation aux examens, on observe encore, comme à l'époque des cours par correspondance, une activité pédagogique basée prioritairement sur la réalisation et l'accompagnent aux exercices d'entraînement à l'examen. Nous notons également l'existence de deux regroupements (temps en présentiel), organisés hors sessions d'examen. Et que depuis six ans, les classes virtuelles ont remplacées les séances de tchat, afin de favoriser la compréhension des contenus et les échanges avec les auteurs des cours. La personnalisation et les interactions sociales sont facilitées, l'utilisation des classes virtuelles vient favoriser le suivi de la formation et permet de mettre de l'humain dans un environnement qui peut être vécu comme technique par les étudiants qui découvrent bien souvent ce qu'est la formation à distance. En rendant les étudiants actifs et acteurs de leur apprentissage par le questionnement sur les cours, les enseignants cherchent à animer des séquences pédagogiques plus dynamiques et efficaces.

Concernant le niveau master un, l'équipe pédagogique a favorisé les temps de travail collaboratif en petits groupes, soit à partir d'études de cas ou de travaux réalisés en commun. Ici aussi, les classes virtuelles ont été systématisées, faisant suite également aux rendez-vous tchat qui existaient précédemment. Par ailleurs, chacun des étudiants est tutoré dans la réalisation de ses productions écrites (travaux collaboratifs, dossier exploratoire de recherche, évaluations). En accompagnant les dispositifs distanciels initiaux, les classes virtuelles constituent une réponse aux questions de lutte contre l'abandon, taux de connexion et de complétude. Le sentiment de solitude que peuvent ressentir les étudiants face aux écrans est réduit, l'animation et l'accompagnement des apprentissages à partir des contenus d'enseignement 
peut plus facilement devenir une réalité. Pour cela, les enseignants sont appelés à changer de posture et à se faire animateurs, ce changement chez des universitaires nécessitent un accompagnement et bien souvent une transformation de leur pratique de la pédagogie.

Enfin, c'est en master 2 que les classes virtuelles ont tout d'abord été expérimentées, dans une logique cherchant à rompre l'éloignement caractéristique de la situation de l'apprenant à distance. II s'agissait en particulier d'accompagner à l'apprentissage de la démarche de recherche. Même si des regroupements en présence ponctuent l'année environ tous les deux mois, il a semblé intéressant de renforcer la présence dans la distance en faisant usage de la téléprésence, conformément à la demande des étudiants. Si dans les premiers temps, il s'agissait surtout de répondre aux questions des étudiants, on observe que de plus en plus ce sont à présent les étudiants qui y présentent leurs travaux. Là encore, les classes virtuelles apportent de l'humain dans les dispositifs en ligne, il favorise à la fois une appropriation des contenus des cours et le renforcement d'une socialisation en formation qui se poursuit au-delà des regroupements en présentiels. C'est d'ailleurs la notion de «regroupements » qui est aussi utilisée, comme étant un rendez-vous à ne pas manquer.

\section{La classe virtuelle, de la métaphore spatiale à la dimension sociale de la classe à distance}

S'interroger sur l'usage du mot « classe virtuelle » oblige à faire référence à l'école (Verquin Savarieau et Daguet, 2016). Pour cette raison, si ses concepteurs ont fait le choix de lui donner ce nom, les enseignants interrogés le remplacent dans leur communication auprès des étudiants ou dans les emplois du temps, par les termes de " séminaires virtuels", " réunions virtuelles " (Wallet, 2012), et même " webinaires " (Power et Desjardins, 2012). Nous remarquons que la comparaison induite par cette appellation de " classe virtuelle » avec la classe traditionnelle, ne convient pas pour les publics adultes en reprise d'études, qui se retrouvent ainsi projetés dans leur situation qui détonne avec les publics habituels de l'université. C'est la raison pour laquelle, le nom choisi par les concepteurs n'est pas celui retenu la plupart du temps par les enseignants. Cependant, la classe virtuelle qui est tout d'abord un dispositif de formation que l'on utilise en entreprises, constitue en enseignement supérieur une modalité qui conduit à privilégier une pédagogie active dans laquelle l'apprenant devient acteur de ses apprentissages. En rejoignant l'étudiant dans ses questionnements, le dispositif classe virtuelle favorise l'échange et le partage et favorise le suivi de formation. Le fait que l'étudiant devienne le centre du dispositif de formation, est à l'université une nouveauté qui implique une transformation de la pédagogie universitaire. Ce changement de paradigme, est dans le cadre de la formation à distance, une modalité qui est fort appréciée des étudiants.

Ainsi, le choix de ce dispositif médiatisé influencerait-il la pédagogie mise en œuvre, afin de permettre de se démarquer de l'enseignement en classe traditionnelle, très fréquemment transmissif, didactique et verbal (Jacquinot-Delaunay, 2002)? La classe traditionnelle en tant qu'espace de socialisation est aussi très souvent, l'espace de la parole du maître dans lequel l'élève apprend à écouter. C'est donc le maître qui donne la parole lorsque l'élève la demande en levant le doigt. II est notable que les concepteurs de la classe virtuelle aient tenu à conserver cette pratique, en introduisant une icône à cet effet. Nous retiendrons que si théoriquement la communication orale spontanée pourrait y avoir lieu, il est manifeste que la bande passante souvent encore trop faible, oblige l'enseignant à gérer l'ouverture des micros pour l'ensemble des acteurs de la formation, en fonction des demandes de prises de parole. C'est donc bien lui qui comme en classe, donne la parole, mais cette possibilité offerte aux étudiants est-elle saisie ou préfèrent-ils se réfugier derrière l'écrit, comme cela se fait parfois dans les cours en présence? Par ailleurs, notons que les étudiants peuvent également interagir par écrit via le forum de discussion ici appelé "conversation», ou bien encore en indiquant leurs réactions ou émotions, au travers de l'affichage d'un 
choix d'icônes sensées faciliter les interactions, dans une application appelée " définir l'état » (lever la main, accepter, refuser, sortir, parler plus fort, moins fort, accélérer, ralentir, rires, applaudir). Les systèmes de communication éducative médiatisée articulent le technique, le social et le symbolique, afin de permettre des interrelations qui nécessitent de repenser la relation pédagogique.

\section{La classe virtuelle un dispositif de communication et de formation médiatisées}

\subsection{De la téléprésence aux questions de médiatisation}

De ce premier constat, les chercheurs ont, dans les travaux sur les technologies éducatives, interrogé les modalités de la prise en charge de l'absence physique. Le texte incontournable de Jacquinot (1993, p. 64) questionne par exemple « la transposition machinale et machinique de l'acte éducatif, à travers divers substituts » qui bien souvent continue de faire référence au modèle paradigmatique du dialogue maîtreélève. Le changement opéré est aussi dans la mutation du rapport au savoir et des supports de transmission de ce savoir. Ainsi Marchand (1998), interroge l'histoire de l'humanité qui est ponctuée par des changements dans ce rapport. Elle questionne l'utilisation des technologies de l'information et de la communication (TIC), le rapport au savoir et aux enseignants, soulignant que les TIC modifient la nature même du savoir qui pourrait devenir, collectif, partagé au sein d'espace en interactions.

Ainsi, les questions de téléprésence, caractéristiques des travaux des années 80 , qui se référaient aux modalités de la présence à distance (Henri, 1989; Lamy, 1985; Feenberg, 1989; Harasim,1990 et Hilt, 1986) ont été remplacées par celles qui interrogent l'interaction pédagogique. II en ressort les nombreux travaux portant sur l'analyse de la médiation et de la médiatisation, où se construisent à la fois les connaissances dans une négociation sociale, et les nouveaux rôles des apprenants et des enseignants (Henri et Kaye, 1985; Henri, 1992; Peraya, 1995, 1999; Peraya et Dumont, 2003; Charlier et al., 2007; McBrien et al., 2009).

Pour Charlier et al., le processus de médiatisation désignera, d'abord en enseignant « le processus de conception et de mise en œuvre de tels dispositifs " (...), processus dans lequel le choix des médias les plus adaptés ainsi que la scénarisation occupent une place importante ". Le processus de médiatisation interroge la mise en œuvre d'une ingénierie de formation et d'un design pédagogique et portent sur deux dimensions du dispositif de formation: le choix des objets et les fonctions qui leur sont attribuées. Concernant le choix de la classe virtuelle synchrone, nous interrogeons l'expérience éducative réalisée à partir de la pédagogie mise en œuvre, au sens de Garrison et Bayaton (1987, p. 14), pour qui le plus important dans la formation à distance n'est pas la non-continuité de la nature de la transaction, mais bien l'expérience éducative elle-même. Pour ces derniers, "le concept crucial et central » en enseignement à distance est « le contrôle ».

Nous retiendrons que la classe virtuelle est un artefact de formation à distance qui permet d'établir des échanges synchrones pouvant utiliser l'image, le son et le texte.

C'est une application de téléconférence assistée par ordinateur (TCAO). Souvent appelée visioconférence, ses usages se sont développés dans les années 90, d'abord dans l'enseignement supérieur puis progressivement dans l'enseignement secondaire et primaire au fur et à mesure que les technologies Internet se sont développées et que leur coût a diminué. (Ferone et Lavenka, 2015, p. 2). 
Pour nous, la classe virtuelle dont l'objet est au départ la possibilité d'organiser des réunions à distance, regroupe des applications synchrones autorisant une transmission audio/vidéo en multipoints, mais aussi le partage et l'échange autour d'un tableau blanc, d'un fichier, d'une recherche en direct sur le web, d'un chat, ou encore des fonctionnalités permettant par exemple le travail en sous-groupes ou la réalisation synchrone de sondages en ligne. Le logiciel de classe virtuelle utilisé dans cette recherche est « Adobe Connect ", que les étudiants appréhendent à partir d'un tutoriel et d'un temps de présentation. Pour les enseignants, s'ils étaient accompagnés au départ par un ingénieur pédagogique multimédia dans leurs premiers pas, c'est surtout en faisant qu'ils se familiarisent avec son usage.

Toutefois, comme l'a souligné Macedo-Rouet (2009, p. 84) dans un article qui porte sur la visioconférence en enseignement, même si elle présente de nombreux avantages, la communication par visioconférence peut y être rendue difficile par des "problèmes spécifiques ", comme des délais de transmission trop lents, une qualité du matériel informatique parfois insuffisante, des prises de paroles difficiles ou bien encore la communication distante qui demande un langage particulier. Ainsi, rejoignant les travaux de Fasso (2013), cette recherche questionne la valeur pédagogique de cette intégration. Pour ce faire, les compétences et les perceptions des technologies par les enseignants nécessitent d'être interrogées.

\subsection{Le travail pédagogique instrumenté entre interactions et interactivités}

Nous basant sur la définition du terme de dispositif proposée par Peraya (1999, p. 153), nous définissons la classe virtuelle comme étant un "dispositif de communication et de formation médiatisées ", soulignant ainsi la double nature communicationnelle et formative de l'outil, soit comme : « une instance, un lieu social d'interaction et de coopération possédant ses intentions, son fonctionnement matériel et symbolique enfin, ses modes d'interactions propres ".

Nous interrogeons la communication médiatisée que l'on peut y observer, soit le " médium ", en tant qu'intermédiaire obligé qui rend médiate la communication entre les interlocuteurs, mais aussi le " média » et le " médiateur ». Communiquer en formation ne consiste donc pas uniquement à transmettre des contenus, mais constitue fondamentalement un acte social qui s'inscrit dans le jeu des interactions. Outre le ton, la formulation, le mode du discours (narratif, descriptif, explicatif, argumentatif), la spécificité de cette communication doit s'adapter aux caractéristiques du public visé.

Par conséquent, loin de constituer une fin en soi, les pratiques pédagogiques instrumentées doivent démontrer les avantages qu'elles permettent. En cela, elles interrogent à la fois l'activité de l'apprenant dans sa construction du savoir et le rôle joué par l'enseignant dans ces apprentissages. Pour Altet (1997, p. 15) : «Ce sont des pédagogies des moyens d'apprendre, de la réussite qui nécessitent la mise en place d'une instrumentation pédagogique et didactique avec médiation de l'enseignant ». De ce fait l'analyse des pratiques pédagogiques instrumentées interroge les composants du dispositif de communication et de formation médiatisées, exercice qui a été initialement effectué par Peraya (1999, p. 158) et que nous avons présenté dans un précédent article (Verquin Savarieau et Daguet, 2016). 


\section{Problématique et méthodologie de la recherche}

\subsection{Problématique et hypothèses de recherche}

Problématique : L'usage des classes virtuelles synchrones modifie-t-elle la professionnalité enseignante et quelles sont les transformations qui en résultent?

H1 : L'usage des classes virtuelles synchrones modifie l'espace d'intervention des enseignants.

H2 : L'usage des classes virtuelles synchrones introduit de nouvelles normes et valeurs pour la profession enseignante.

\subsection{Méthodologie de la recherche}

\subsubsection{FAIRE DIRE, L’ÉLABORATION D’ENTRETIENS SEMI-DIRECTIFS}

Notre méthodologie de recherche est double : d'une part des entretiens réalisés auprès d'enseignants du département des sciences de l'éducation et d'autre part une analyse des différentes interactions observées à partir de l'enregistrements de quelques classes virtuelles. Les entretiens semi-directifs ont été construits autour d'un guide permettant aux enseignants usagers des classes virtuelles synchrones d'évoquer dans un premier temps leurs rapports et usages des technologies. Dans un second temps nous leur avons demandé d'évoquer leurs pratiques de ces classes virtuelles. Notre guide a été élaboré dans une posture très proche de la non-directivité au sens rogérien du terme (Blanchet et al., 2013; Blanchet, 2003; Ghiglione et Matalon, 1998). Nous cherchons avant tout à observer au-travers de celui-ci, les représentations des enseignants sur leur rapport général aux technologies et à la classe virtuelle synchrone en particulier. Ainsi, nous avons délibérément fait le choix de ne pas construire d'outil qualitatif directif ou semi directif fondé sur une échelle mesurant a priori le degré d'usage des acteurs. En effet, nous cherchons avant tout à observer au travers des discours tenus, les représentations du métier d'enseignant et des technologies, mais aussi ce qui nous pouvons appeler l'expérience vécue des évolutions du métier à partir de l'intégration des technologies, en particulier celle réalisée en classes virtuelles synchrones. L'autre versant de notre protocole concerne l'analyse interactionnelle d'un échantillon de classes virtuelles. Notre intention est qu'au travers de ces observations nous puissions confronter « le faire et le dire », en d'autres termes quel est l'écart des pratiques des enseignants observés et leurs déclarations.

Nous avons retenu dans notre guide d'entretien, trois axes nous permettant de mieux appréhender le degré d'usages des technologies de ces enseignants et en particulier de la classe virtuelle :

- $\quad$ Leurs usages personnels des technologies (ordinateur, internet, Smartphones...),

- $\quad$ Leurs usages professionnels des technologies (préparation, pendant, après les cours ou encore pour ou avec les étudiants),

- $\quad$ Leurs usages des classes virtuelles et notamment de ses fonctionnalités avancées.

Nous considérons que la professionnalité d'un enseignant est inséparable de sa professionnalisation, soit comme Wittorski (2008, p. 24) le précise, reprenant Le Boterf (2007), de son itinéraire professionnel comportant à la fois, son histoire, sa socialisation, les situations professionnelles qu'il a rencontrées et des situations et parcours de formation variés qui constituent autant d'espaces dans lesquels le sujet déploie une activité propice à son développement, entendu ici sous le double aspect des compétences et des identités. L'itinéraire de professionnalisation peut être plus ou moins institutionnalisé ou programmé 
d'avance, dans tous les cas, il engage l'individu dans des séries d'expériences qui sont sources potentielles du travail de leur professionnalité. Etudier l'intégration des TIC et la professionnalité enseignante consistera donc en creux, à étudier le rapport des enseignants aux technologies et à mesurer la façon dont ils les intègrent dans leur travail.

Pour ce faire, nous devons distinguer deux sens au mot « expérience » :

1) L'expérience au sens « d'avoir de l'expérience » est celle déjà constituée, stabilisée, voire non modifiée depuis un certain temps. Cette expérience sera donc celle d'un cadre structurant et rassurant et s'apparente à une durée. Seront alors observés dans ce cas, les ingrédients qui constituent les fondements de l'activité, stabilisés et caractéristiques de la professionnalité.

2) L'expérience au sens de "faire l'expérience de » correspond pour une personne à la découverte de quelque chose de nouveau. Cette expérience " découverte », transformatrice de l'activité, consistera dans une rupture des habitus, une remise en cause des choses acquises. Seront alors observées les métamorphoses de la pratique qui constituent un temps d'instabilité et de réappropriation de l'activité.

Le développement de l'expérience occupe une place importante dans l'intégration des TIC, mais toute la difficulté réside bien souvent dans le fait que cette découverte s'effectue en situation réelle de travail. Se pose alors les questions de la préparation ou de la formation à la variété des situations auxquelles l'enseignant pourra être confronté. L'expérience s'inscrit alors dans ce processus de transformation de l'être, dans ce sens qu'elle peut être définie comme étant ce que je fais de ce qui m'advient. Le sujet doit élaborer son expérience à partir de l'activité qu'il a réalisée. Barbier $(2013$, p. 73$)$ définit

"L'élaboration de l'expérience comme la construction par un sujet, pour ses actions en cours et à venir, et à partir d'épisodes antérieurs de son activité, de constructions mentales, rétrospectives et anticipatrices d'unité de sens, parce qu'ordonnées autour de ses intentions personnelles de transformation du monde».

L'expérience est une et se poursuit d'une activité d'apprentissage à l'autre. Ainsi, les enseignants s'appuient sur l'expérience professionnelle qu'ils ont acquise, ils parlent beaucoup plus facilement en personnes qui pratiquent une technique, en « praticiens » autrement dit, et cette expérience peut, comme l'affirme Pastré (2013, p. 97), les enfermer « dans la complexité d'une pratique, mais aussi dans la singularité d'une situation ».

À la suite des nombreuses recherches consacrées au rôle de l'expérience dans l'apprentissage des adultes qui ont fait l'objet d'une revue de littérature (Balleux, 2000), notons que l'activité n'aura pas toujours la même valeur: certaines seront significatives car remarquables par le sujet et possiblement transformables en expérience vécue, tandis que d'autres seront non significatives et seront désignées par "activités indifférenciées ". L'apprentissage de l'usage des classes virtuelles peut alors rejoindre la question de la transformation de cette activité indifférenciée et du vécu en " expérience ressource " pour l'action (Zeitler, 2013, p. 137). L'expérience dont nous parlerons ici est celle de processus d'apprentissage et de développement de la personne. Pour résumer, l'expérience est alors présentée comme l'ensemble des opérations mentales et actionnelles qui permettent la transformation de ressources pour l'activité de la personne, y compris pour les activités futures (Balleux, 2000). Ainsi, nous retiendrons qu'en français, il n'existe qu'un seul mot, " expérience ", pour désigner deux sens distincts, alors que l'anglais en offre deux (to experiment, to experience). Ces deux sens " expérimentation " et " expérienciation ", sont combinatoires. Le premier est un fait observé, soit une observation dans l'intention d'étudier un phénomène (Kohn et Nègre, 2003, p. 85), « d'aller voir de qui en est réellement plutôt que de spéculer sur un phénomène ", soit l'expérimentation pour signaler le recours à un mode d'expérience dans une visée scientifique. Le deuxième, est l'expérience comme le fait vécu et éprouvé, souvent considéré comme un 
élargissement ou un enrichissement de la connaissance, du savoir. Ces deux dimensions de l'expérience sont donc combinatoires : tandis que l'une porte sur l'observable, l'autre s'intéresse à l'observateur.

\subsubsection{MODALITÉS DE L’ANALYSE DU CORPUS}

Nous pouvons donc logiquement supposer, dans une perspective d'analyse à grain moyen, que c'est dans la rencontre de situations professionnelles inédites, lieu d'expression de l'expérience (sens 2), que la professionnalité se trouve reconfigurée car retravaillée par les individus. Ces reconfigurations de la professionnalité des enseignants ayant pris en charge des cours dans lesquels sont introduites des classes virtuelles, sont donc analysées à partir d'expériences réalisées et décrites pendant les entretiens. Pour cela, nous avons constitué un échantillon de 12 enseignants-chercheurs qui sont des usagers des classes virtuelles synchrones au sein de nos dispositifs L3 et M1 à distance. Nous avons retenu deux variables pour l'analyse de notre corpus :

- V1: L'Expérience en enseignement supérieur. Nous catégorisons le sujet parmi les " confirmés » s'il enseigne depuis plus de 10 ans à l'université. Par ailleurs, il est à noter qu'en sciences de l'éducation, les enseignants-chercheurs ont très souvent une expérience préalable de l'enseignement, que ce soit en primaire, secondaire, ou en formation d'adultes. De même nous catégorisons les sujets comme "débutants » quand ils possèdent une faible expérience dans l'enseignement universitaire, mais ils peuvent avoir enseigné par ailleurs.

- V2 : Le rapport aux technologies. Cette variable concerne l'appétence pour les usages des technologies de l'information et de la communication. Notamment, pour ce que Dimet (2004) présente comme la "bureautique professorale " dès le milieu des années 1990, à savoir les usages hors classes, souvent dans le but de préparer les cours mais également les usages administratifs et évaluatifs hors la salle de cours.

Les enseignants qui ont une appétence sont ceux qui disent apprécier utiliser régulièrement les technologies en cours. À contrario, ceux qui ont une faible appétence pour les technologies sont comparables à ceux décrits par Rinaudo et Ohana (2007) quand ils dressent une typologie d'enseignants usagers du dispositif « Ordi 35 », les « malgré eux ». Dans le cadre des dispositifs à distance qui ont servi de terrains à cette recherche, les enseignants qui relèvent de cette deuxième catégorie utilisent les classes virtuelles synchrones parce que c'est une composante technologique du dispositif mais, contrairement aux appétants, ils y sont contraints et ne vont chercher à utiliser des fonctions avancées de la classe virtuelle, comme le module de QCM en ligne. Nous pensons donc qu'en creux, étudier le rapport aux technologies des enseignants permet de mieux comprendre les choix qu'ils effectuent dans la manière dont ils intègrent les usages des technologies dans leurs pratiques pédagogiques. L'échantillon peut donc se résumer comme suit : 
Tableau 1

Description de l'échantillon

\begin{tabular}{|c|c|c|}
\hline $\begin{array}{l}\text { V1: Expérience d } \\
\text { V'enseignemer } \\
\text { Technologies }\end{array}$ & Confirmé & Débutant \\
\hline $\begin{array}{l}\text { Forte appétence pour les } \\
\text { usages des technologies }\end{array}$ & 3 & 3 \\
\hline $\begin{array}{l}\text { Faible appétence pour les } \\
\text { usages des technologies }\end{array}$ & 3 & 3 \\
\hline
\end{tabular}

L'analyse de contenu est principalement thématique catégorielle et construite en nous fondant sur Bardin (2007) ou encore Ghiglione et Blanchet (1991). Les indicateurs permettant d'analyser l'intégration des classes virtuelles ont été construits au travers des questions et relances portant sur les usages de ces classes tels qu'ils nous ont été décrits par les enseignants.

\section{Présentation de quelques résultats}

\subsection{Dans la classe, hors la classe : l'évolution de l'espace professionnel des enseignants}

Nos interlocuteurs expriment unanimement leur étonnement de pouvoir réaliser leurs cours depuis chez eux. Pourtant aguerris à la formation en ligne, le fait de donner à voir leur " chez eux " constitue pour chacun un changement qui interroge. L'expression " télétravail » est même prononcée, soit comme la faculté de mener son activité d'enseignement en dehors d'une situation accoutumée, marquant là la séparation avec l'institution. (11): "C'est globalement toutes les questions comme pour le télétravail. Globalement on a l'intimité. C'est-à-dire que les étudiants quelque part, ils voient chez toi ! ». (I2) : " C'est quand même assez déstabilisant d'être au boulot alors qu'on est chez soi, sur des créneaux horaires pas habituels ».

Derrière les questions d'intimité, c'est aussi les signes de l'institutionnalisation et du professionnalisme qui sont interrogés, deux de nos interviewés ayant ressenti la nécessité de positionner derrière eux une bibliothèque et un troisième, après avoir imaginé un " drap estampillé du logo du campus Forse " (comme c'est le cas de la salle de visioconférence de l'université), s'est positionné au dos de son canapé, soit dans un environnement neutre.

(I2) : « Je me mets dans mon bureau chez moi. Derrière il n'y a que des bouquins. Donc ça fait très sérieux. Par ce qu'il faut faire attention à tout. On est quand même chez soi quoi ! Donc ça pose problème aussi... Problème d'intimité. ".

(14) : "Partager la vidéo c'était rentrer dans ma chambre. Donc je me dis tu as toujours ta bibliothèque derrière toi ». 
Apparaît ainsi, la nécessité de se référer à un espace qui n'est pas celui de la maison, dans une logique de rupture ou de passage par rapport à environnement ambiant, comme l'indique très explicitement (I3) :

"Il est nécessaire de construire un petit rituel, un cadrage pour que depuis chez soi, l'enseignant puisse créer cette structure et rentrer dans son rôle, en quelque sorte. Sortir de son espace intime et rentrer dans un espace public. C'est comme si on amenait nos étudiants dans notre salon. II n'y a pas de rupture entre vie privée et vie professionnelle. On est dans un salon et d'un seul coup on rentre dans une classe $"$.

Enfin, ce qui est classiquement signalé pour les étudiants à distance, est également interrogé ici, soit l'organisation familiale, notamment le non-partage d'un bureau, d'un réseau internet ou d'un ordinateur, durant le temps de la classe virtuelle. Nous avons l'impression que certains lieux et le réseau informatique en particulier, sont au moment de la classe virtuelle sanctuarisés, lorsque les autres membres de la famille sont présents. (I1) affirme;

"Il y a des moments on ne peut pas parler à la maison! En tout cas on ne fait pas les clowns à ce moment-là ! On sait qu'il y a un truc particulier à la maison ». (I2) : "Ce soir personne dans la maison utilise l'ordi sauf moi ». (I3) : "Alors j'ai fait en sorte à chaque fois de, de n'avoir personne chez moi le jour J. Moi j'ai un deux-pièces. S'il y avait des gens ça se verrait tout de suite ».

\section{2 La distance/proximité de l'expérience professionnelle vécue}

Sont ici mises en évidence les contraintes techniques liées aux environnements médiatisés d'apprentissage, mais surtout à la non-maîtrise de l'ensemble des applications offertes par l'outil, mentionnée par nos interlocuteurs. Cette expérience de la non-infaillibilité de l'outil et de son utilisateur rappelle, s'il en est nécessaire, qu'il ne s'agit pas d'une pratique magique mais bien d'un exercice qui nécessite un entraînement et le développement de nouvelles compétences. (I2) affirme par exemple :

«Une fois je n'avais pas de réseau. Mais en même temps, quand je dépends de la technique ça m'angoisse. Donc, si je ne suis pas au rendez-vous parce que la technique ne marche pas je suis vraiment très déstabilisée !»

D'autres contraintes exprimées sont parfois incontournables car faisant parties de la conception de l'outil, comme c'est le cas ici : (I3) « J'aimais bien l'idée qu'ils pourraient prendre la parole quand ils le veulent, mais sans lever la main. " Nos interlocuteurs expriment très clairement deux choses quant à la nature de la communication médiatisée caractéristique de la classe virtuelle. Les nombreuses possibilités de communication que nous avons précédemment décrites semblent constituer pour trois d'entre eux plutôt une difficulté, face aux flux des informations à traiter (visuels et auditifs), mais ces propos sont également à mettre en relations avec une non-maîtrise affirmée de l'outil. (I3) :

"On se dit du jour au lendemain vous passez à l'écran vous devez gérer le live. Je trouve qu'il y avait beaucoup de choses à gérer ça m'a permis de me concentrer sur ma voix et les documents à partager ».

Il est également intéressant de souligner que la possibilité offerte de pouvoir s'exprimer oralement, constitue une véritable satisfaction exprimée à part deux d'entre eux, comparativement à l'usage du tchat. Nous retiendrons deux expressions, pour la première, (I1) : «Ce n'est plus la médiation par le clavier, c'est la médiation par le langage ", pour la seconde, (I2) : " Je préférais parler plutôt que de taper sur une machine ॥. Le langage écrit est ici attribué au clavier ou à la machine plutôt qu'à celui qui en rédige le contenu pour près de la moitié de nos interlocuteurs. La communication médiatisée en classe virtuelle est- 
elle plus aisée comparativement à d'autres usages, comme c'est le cas du tchat, puisqu'elle semble parfois se faire oublier?

Ainsi, le changement en cours oblige à se remémorer d'où l'on est parti, les conditions d'émergence et le parcours entrepris. Il est à noter que nombreux sont les enseignants qui utilisent aujourd'hui les classes virtuelles et qui participaient jadis aux séances de tchats synchrones. Cette proximité de l'expérience de la synchronie dans un dispositif médiatisé semble les rassurer, mais n'est pas sans interroger le choix de la temporalité retenue. S'il s'agit ici d'une recherche d'une vraie proximité avec les étudiants, pourquoi la synchronie représenterait-elle une innovation plutôt que des échanges asynchrones?

\subsection{Les savoirs pluriels et multimodalité, intervenir sous pression?}

L'appropriation d'une pratique instrumentée nécessite du temps. Plusieurs classes virtuelles sont nécessaires pour s'adapter à la pression liée à l'intervention synchrone médiatisée. Ajoutons à cela la nécessaire formation de l'enseignant, qui prend la forme au démarrage d'une coanimation avec un informaticien. Sans un véritable travail autour de l'analyse des pratiques menées, il nous semble que l'apprentissage qu'elle nécessite est forcément long, d'où le fait que nous observons que l'ensemble des applications des classes virtuelles sont loin d'être employées. L'intégration d'une pratique instrumentée nécessite de repenser l'ensemble du dispositif où elle intervient, ainsi que l'organisation de l'enchevêtrement des temps synchrones et asynchrones du processus formation et du travail personnel et/ou collaboratif des étudiants. À de nombreuses reprises, nos interlocuteurs ont souligné qu'en direct, aucune perturbation n'est acceptable, sous peine d'une perte de contrôle de la classe virtuelle, mais qu'ils apprenaient à faire face à l'imprévu et à connaître les applications qu'offre ce dispositif.

Ici le lieu réservé à la pédagogie, même s'il empiète sur l'espace intime, ne semble pas très intimiste. II est fréquent que l'entourage vienne voir ce moment d'exception, à commencer par les enfants. Comment dans ce cas l'étudiant peut-il exprimer ses difficultés d'apprentissage? Pourtant les étudiants prennent la parole, non pas comme une parole scolaire mais pour faire part de leurs émotions et exprimer leurs accords ou réticences face à tel ou tel point du contenu proposé. L'étudiant par sa prise de parole devient alors coconcepteur des ressources d'apprentissage, comme l'ont déjà exprimé Develay et Godinet (2007), en cela les pratiques instrumentées responsabilisent et nécessitent une relation privilégiée avec l'enseignant. Ce dernier peut alors dans le meilleur des cas questionner, porter l'intérêt du groupe sur ce qu'il considère comme fondamental : argumenter et négocier, au sens de la régulation. II se fait alors animateur et quitte parfois son identité d'enseignant afin d'assurer la plus grande participation de chacun; cela ne se fait pas sans se rendre plus proche et plus disponible à « ses hôtes ».

Nos interlocuteurs retiennent en particulier la possibilité offerte de pouvoir s'exprimer oralement, ce qui constitue une véritable source de satisfaction pour la moitié d'entre eux, comparativement à l'usage du tchat qui prévalait auparavant. Nous retiendrons deux expressions, pour la première, (I1) : "Ce n'est plus la médiation par le clavier, c'est la médiation par le langage », pour la seconde, (I2) : " Je préférais parler plutôt que de taper sur une machine ". Le langage écrit est ici attribué au clavier ou à la machine plutôt qu'à celui qui en rédige le contenu. Cela pourrait-il signifier que pour la moitié de nos interlocuteurs, la communication médiatisée en classe virtuelle est plus aisée comparativement à d'autres usages (tchats), puisqu'elle semble parfois se faire oublier? II semble par ailleurs que les possibilités importantes d'interactions en communication médiatisée accélèrent le rythme des échanges et la nécessité d'être réactif. Se pose au final de façon accrue la question du cadre d'intervention et du contrôle du dialogue afin qu'il puisse être source d'apprentissage. 


\subsection{La médiation langagière : le corps instituant de l'enseignant}

Bien que dispensée à la maison, la classe virtuelle est un espace qui diffuse les signes propres de l'institution universitaire et de la professionnalité enseignante. Nos résultats montrent à quel point nos interviewés ont été perturbés par le fait de donner à voir leur chez eux. De même le fait de se donner à voir, nécessite de préparer son corps en se maquillant par exemple, comme c'est le cas de cette interlocutrice :

«L'espace privé c'est... faire attention... C'est comme si on allait travailler... Il y a des jours où je ne fais que de la recherche... Bon, bah, je n'ai pas besoin de me faire une beauté. De faire attention à mon look. Je suis chez moi quoi ! Et là, c'est quand même assez paradoxal. C'est deux logiques contraires. C'est-à-dire que je suis chez moi, mais je ne suis pas chez moi ! C'est-à-dire que je suis dans un espace privé, mais c'est quand même un espace public parce que je me donne à voir. Et, je me donne à voir chez moi ! Donc ça suppose une préparation préalable, d'abord psychologiquement parce que... se faire une beauté, se préparer à une interaction avec des publics..." ".

Cette « mise en scène de la vie quotidienne », pour reprendre les travaux de Goffman (1973), renvoie aux rites de présentation de soi. Intervenir en classe virtuelle, c'est se mettre en scène, afin de chercher à construire dans la distance une relation sociale, une sorte de représentation dont le but est de répondre aux besoins d'un public dont on va se mettre à l'écoute. Le dialogue avec ce public renvoie à une mise à l'épreuve, celle de savoir établir des rapports d'intimité complices et de partages, afin que les étudiants osent s'exprimer, y compris s'il s'agit de difficultés ou de malaises face à des incompréhensions.

L'importance du corps instituant et parlant de l'enseignant est donc à interroger, car en effet, si comme l'affirme Jorro (2006, p. 6) : "Le corps parlant de l'enseignant semble faire la différence en situation didactique ». La reconnaissance de l'expression des corps, au travers des gestes ou de l'expression physique de l'enseignant ou de l'étudiant, qui traditionnellement suscite une lecture permanente en situation de face à face pédagogique, est en classe virtuelle à requestionner. Les gestes des enseignants peuvent notamment structurer les échanges, devenir parfois des gestes de planification ou de remédiation, d'accueil, d'écoute et de reconnaissance; or, nous savons qu'en classe virtuelle, le langage est l'instrument principal de l'enseignant et s'exprime presque sans gestes langagiers apparents. Est-ce concevable de passer tous ces gestes qui font partie des habitus d'apprentissage par écrit? Observe-ton par exemple l'utilisation de smileys? Nous notons que les étudiants ressentent le besoin de voir le visage de l'enseignant à l'écran et qu'à l'inverse, les enseignants se plaignent ne pas voir les étudiants. C'est donc sur toute l'organisation de l'environnement visuel sur lequel va porter l'attention de l'enseignant, qu'il s'agisse de son propre corps (maquillage, apparence), alors même qu'on ne le verra pas obligatoirement, ou du décor qu'il donnera à voir (environnement physique) et design de ses supports d'intervention (Verquin Savarieau et Daguet, 2016).

L'appropriation d'une pratique instrumentée nécessite du temps. Plusieurs classes virtuelles sont nécessaires pour s'adapter à la pression liée à l'intervention synchrone médiatisée. Ajoutons à cela la nécessaire formation de l'enseignant, qui prend la forme au démarrage d'une coanimation avec un informaticien. Sans un véritable travail autour de l'analyse des pratiques menées, il nous semble que l'apprentissage qu'elle nécessite est forcément long, d'où le fait que nous observons que l'ensemble des applications des classes virtuelles sont loin d'être employées. L'intégration d'une pratique instrumentée nécessite de repenser l'ensemble du dispositif où elle intervient, ainsi que l'organisation de l'enchevêtrement des temps synchrones et asynchrones du processus formation et du travail personnel et/ou collaboratif des étudiants. L'une de nos interlocutrices affirme : 
"Déjà, à l'origine des classes virtuelles, il y a les tchats le soir entre 21 heures et 22 heures qui portaient sur le cours. (...) Bien souvent on avait quelques questions à l'avance, via le forum, pour organiser notre tchat d'une heure sur le cours et puis la technologie ayant avancé, ça s'est transformé en classe virtuelle de façon facultative, mais j'ai tout de suite accepté parce que le tchat me plaisait moyen. Je préférais parler plutôt que de taper sur une machine ».

Cette description très imagée de l'usage des classes virtuelles et intéressante, car on le constate, ici c'est la possibilité d'échanger oralement avec les étudiants qui la motive; par ailleurs la classe virtuelle ne constitue qu'une partie du dispositif de formation médiatisée existant; elle est utilisée en lien avec le contenu d'un cours préalablement déposé sur une plateforme et un forum dans lequel il est possible de déposer " des questions à l'avance ».

Un autre raconte :

"Pour ma première classe virtuelle j'avais 40 étudiants. Donc c'était ma première et c'était trop déroutant pour moi. Par contre, la deuxième j'ai essayé un peu d'alterner différents médias donc diaporama, vidéo, questions-réponses, j'ai essayé d'utiliser les outils disons le sondage je me rappelle les pourcentages qui s'affichaient et la troisième j'étais en co-animation avec un collègue. Là j'étais plus à l'aise pour essayer justement toutes les possibilités que j'ai découvertes avec Connect ».

Ce qui ressort de cette affirmation semble être l'insécurité ressentie pour une enseignant qui utilise la classe virtuelle seul pour la première fois. Le manque d'accompagnement dans une situation réelle de travail interroge le ressenti des étudiants qui semblent jouer le rôle de cobayes.

\section{Retour sur les hypothèses}

Concernant l'hypothèse H1 L'usage des classes virtuelles synchrones modifie l'espace d'intervention des enseignants, nous pouvons affirmer qu'elle est validée. En effet, comme nous venons de l'illustrer, l'usage des classes virtuelles modifient l'espace professionnel des enseignants, en introduisant à domicile des temps d'enseignement. Si ce constat, d'une interpénétration entre l'espace du travail et le domicile peut être fait aujourd'hui, pour de nombreuses professions, elle constitue dans le cas des enseignants, une profonde mutation. En ce sens que la salle de classe ou de cours, a jusqu'à aujourd'hui représentée un espace d'intimité, un lieu de vie dans lequel il est possible d'apprendre la socialisation. Cette socialisation manifeste l'ensemble des mécanismes par lesquels un individu intériorise les normes et les valeurs d'un groupe d'appartenance et construit son identité sociale. Cette virtualisation de l'espace classe nécessite donc de repenser cette socialisation à l'aide des réseaux du web 2.0 et interrogent les relations que l'on peut y entretenir. Elle questionne la mise en scène numérique de soi et l'interpénétration de l'espace privé et de l'espace public. Cette mise en scène interroge la théâtralisation des cours, au sens de ce que les enseignants donnent à voir, d'eux et/ou de leur environnement.

Concernant l'hypothèse $\mathrm{H} 2$ L'usage des classes virtuelles synchrones introduit de nouvelles normes et valeurs pour la profession enseignante, il nous semble que oui, mais le processus de développement est en cours et donc nous manquons de recul sur les effets réels de cette intégration. II nous semble toutefois notable que les changements dans l'agir professionnel des enseignants qui ont été interrogés sont complexes à analyser et nombreux. Nous retiendrons toutefois, qu'aucun des enseignants interrogés n'a continué sa pratique ancienne du chat, même si les effets induits par l'intégration des classes virtuelles semblent induire quelques perturbations dans l'exercice de leur activité enseignante. Si notre terrain de recherche est un dispositif de formation à distance qui existe depuis plus de quinze ans et induit par 
conséquent, une familiarité des enseignants avec les modalités à distance, il interroge les valeurs accordées à la communication médiatisée en enseignement. II questionne également les conditions de la valorisation, voire la reconnaissance des compétences acquises et la capacité de l'université à accompagner l'innovation. Loin des prédictions alarmistes : "Les universités sont comme ces étoiles qui brillent encore mais qui sont mortes depuis longtemps " (Serres, 2003), il semble que les enseignants du supérieur mettent en œuvre de nouveaux moyens pour accompagner les nouveaux publics de l'université. Mais les effets différés dans le temps de l'exercice professionnel à partir des classes virtuelles (elles ont souvent lieu à partir de 20h30), et donc un effet « cours du soir » auquel s'ajoute le sentiment de la mise en place d'une pratique de télétravail interroge la reconnaissance de cette activité professionnelle qui semble encore pour certains relever d'un " excès de zèle ».

\section{Conclusion}

Nous retiendrons des éléments mettant en évidence des changements dans l'agir professionnel enseignant, répondant à trois niveaux de pratique clairement réinterrogés par l'usage des classes virtuelles synchrones. Ces angles de saisie déterminent les processus de reconnaissance de la professionnalité (Jorro, 2006).

\section{1) Le niveau institutionnel ou l'approche macro de la professionnalité enseignante}

Ce niveau est ici en tension avec une réalité plus personnelle, voire intimiste de l'enseignant, qui est appelé à donner à voir son domicile et à se donner à voir dans sa fonction institutionnelle depuis chez lui. Pour cela, le corps instituant de l'enseignant est réinterrogé par le changement survenu à partir de la médiation langagière et de la virtualisation du corps. Le corps de l'enseignant reste un corps instituant mais peu lisible, alors que les enseignants restent sensibles au fait de devoir se mettre en scène en tant qu'enseignants. Avec le corps, c'est aussi la parole instituante qui est en classe virtuelle centrale : il s'agit de rejoindre les étudiants là où ils se trouvent. La classe virtuelle pourrait alors être un instrument de réinstitutionnalisation de la formation, dans le sens qu'elle favorise peut-être l'utilisation par les étudiants des réseaux sociaux mis à leur disposition par l'institution universitaire (forum, classes virtuelles), plutôt que ceux existant à l'extérieur de l'institution universitaire.

\section{2) Le niveau de l'animation de la classe virtuelle ou l'approche méso de la professionnalité}

L'enseignement au sens de "faire cours" se transforme au gré des expériences vécues avec les étudiants. II s'agit alors plutôt d'intervenir en classes virtuelles en ayant à l'esprit de ne pas séparer l'acte d'enseignement de celui de l'apprentissage. La conception de l'enseignement et du rapport au savoir s'en trouve profondément modifiée : il ne s'agit plus d'enseigner dans une optique transmissive, dans une approche descendante ou prescriptive; il ne s'agit plus non plus d'attendre que les étudiants apprennent dans une logique d'ingurgitation ou de soumission à la parole du maître, mais d'interagir avec eux et de chercher à répondre à leurs besoins. La classe virtuelle est donc appréciée comme un espace transactionnel, dans lequel la pédagogie active est recherchée, mettant en évidence le caractère personnel de l'apprentissage et la nécessité d'un feedback par rapport aux activités des étudiants. L'étudiant de son côté, par sa prise de parole devient alors coconcepteur des ressources d'apprentissage, comme l'ont déjà exprimé Develay et Godinet (2007), en cela les pratiques instrumentées responsabilisent et font apparaître une relation renforcée avec l'enseignant. Ce dernier peut alors dans le meilleur des cas, questionner, porter l'intérêt du groupe sur ce qu'il considère comme fondamental : argumenter et négocier, au sens de la régulation. II se fait alors animateur et quitte parfois son identité d'enseignant afin d'assurer la plus grande participation de chacun; cela ne se fait pas sans se rendre plus proche et plus disponible à " ses hôtes". 


\section{3) Le niveau de l'action située ou l'approche micro de la professionnalité}

L'agir professionnel de l'enseignant se trouve profondément remanié en termes de savoirs à mobiliser. Outre les dimensions pédagogiques et disciplinaires, ici la connaissance de l'instrument et plus largement des TIC est incontournable. II s'agit par là-même d'une intégration/actualisation des compétences qui accompagne le changement au travers de savoirs pluriels. Dans ce dispositif synchrone, soulignons la nécessité de contrôler la temporalité, ce qui consiste en : 1) respecter les horaires fixés; 2) faire face en direct à d'éventuels dysfonctionnements; 3 ) tenir ses objectifs pédagogiques tout en réagissant en direct à toute sorte de questions. C'est également tant au niveau technique qu'humain, s'habituer à la pression générée par la conjonction de la synchronie et de la multimodalité (nombre d'applications, documents et interactions à gérer simultanément, communication simultanée à l'écrit et à l'oral), ce que Develotte et Mangenot (2010, p. 6) qualifient de « pression temporelle ».

Ainsi, à l'instar des travaux de Choplin et al. (2007), nous pensons également qu'interroger la professionnalité enseignante et l'innovation pédagogique pousse à porter un regard sur la diversité des expériences observables; au-delà d'études de cas, qu'y a-t-il de généralisable et jusqu'à quel point? C'est également réinterroger les dimensions collectives du travail enseignant, les dimensions espace-temps de la pratique, les dynamiques en cours (historiques et épistémiques), les identités et les compétences en développement, sans oublier les valeurs et les normes propre à chaque métier, mais aussi discipline enseignée. Cela ne peut se faire sans prendre en compte également les évolutions institutionnelles en cours, les publics étudiants qui se diversifient, que ce soit dans leur manière d'apprendre que dans leurs profils sociologiques. Entre logiques d'adaptation et logiques innovantes, les motivations des enseignants dans l'usage des technologies restent à questionner, tout comme l'expérience qui en résulte côté étudiant, que l'on peut interroger en termes de qualité des apprentissages mais aussi d'appropriation de savoirs transférables à la sphère professionnelle, quand il s'agit, comme c'est le cas ici très fréquemment, de publics enseignants ou de formateurs.

Enfin, il semblerait également que le travail de l'enseignant s'intègre véritablement à celui d'une équipe pédagogique, aux professionnalités diverses. L'observation de l'appropriation de l'usage d'une TIC permet de mettre en évidence les évolutions de la professionnalité enseignante. En cela l'avenir de «l'université numérique » n'est pas sans interroger la reconnaissance et l'intégration des pratiques pédagogiques instrumentées dans un univers institutionnel en constante transformations (Baron, 2010). Ce que nous observons ici a pourtant son importance, puisque bien plus qu'une simple médiation, il s'agit à présent plutôt d'une remédiation technologique ayant des effets bien au-delà de la simple sphère de la formation à distance (Poellhuber et al., 2012). Elle questionne d'une manière nouvelle la pédagogie, y compris celle menée en présentiel et la prise en compte des étudiants qui implique une rupture avec les pratiques scolaires, accompagnant par là-même les nouveaux publics des universités. Les pratiques instrumentées telle que celle de la classe virtuelle en petits groupes, comme c'est le cas pour les enseignants interrogés dans cette recherche, permettent de concilier massification et pédagogie différenciée propre à la recherche de la construction des savoirs; elles peuvent devenir des outils précieux dans la lutte contre le décrochage ou l'abandon des étudiants. Le titre par conséquent de " maître de conférences » n'a jamais été aussi impropre aux vécus observés de certains universitaires; ils deviennent des maîtres en diversification, adaptation et éducabilité, tout en cherchant à ne pas perdre la cohérence propre à chaque groupe d'étudiants. 


\section{Liste des références}

Altet, M. (1997). Les pédagogies de l'apprentissage. PUF.

Balleux, A. (2000). Évolution de la notion d'apprentissage expérientiel en éducation des adultes : Vingt-cinq ans de recherche. Revue des sciences de l'éducation, 26(2), 263-286.

Barbier, J.-M. (2013). Expérience, apprentissage, éducation. Dans L. Albarello, J.-M. Barbier, E. Bourgeois et M. Durand, Expérience, activité, apprentissage (p. 65-85). PUF.

Bardin, L. (2007). L'analyse de contenu. PUF.

Baron, G.-L. (2010). Quelles évolutions des professionnalités dans le contexte de l'enseignement supérieur en ligne? Quelques réflexions. Distances et Savoirs, 8(2), 193-205.

Béziat, J. (2012). Formateur en ligne: Vers un modèle d'action: Distances et médiations des savoirs, 2012(1). https://doi.org/10.4000/dms.2519

Blanchet, A. (2003). Dire et faire dire. L'entretien. Armand Colin.

Blanchet, A., Ghiglione, R., Massonnat, J. et Trognon, A. (2013). Les techniques d'enquête en sciences sociales. Dunod.

Charlier, B., Deschryver, N. et Peraya, D. (2007). Apprendre en présence et à distance: Une définition des dispositifs hybrides. Distances et Savoirs, 4(4), 469-496.

Choplin, H., Audran, J., Cerisier, J., Lemarchand, S., Paquelin, D., Simonian, S., ... Jacquinot, G. (2007). Quelle recherche sur et pour l'innovation pédagogique. Distances et savoirs, 5(4), 483-505. https://doi.org/10.3166/ds.5.483-505

Deceuninck, J. (2007). Les campus numériques en France : Réalisations, dynamiques et émergences. Études de communication, (Numéro spécial), 173-192. https://doi.org/10.4000/edc.617

Develay, M. et Godinet, H. (2007). Éléments pour une problématique du changement. Dans J. Wallet, Le campus numérique FORSE: Analyses et témoignages. Mont-Saint-Aignan: PURH.

Develotte, C. et Mangenot, F. (2010). Former aux tutorats synchrone et asynchrone en langues. Distances et Savoirs, 8(3), 345-359.

Daguet, H. et Savarieau, B. (2014). Intégrer les classes virtuelles synchrones à l'université, simple évolution des pratiques ou mutation pédagogique ? frantice.net, 8. http://www. frantice.net/index.php?id=853

Dimet, B. (2004). Enseignants et ordinateurs à l'aube de la révolution Internet. Le cas de l'académie d'Amiens 1980-1997. L'Harmattan.

Fasso, W. (2013). First Year Distance Transition Pedagogy: Synchronous online classrooms. The International Journal of the First Year in Higher Education, 4(1). 33-45. https://doi.org/10.5204/intifyhe.v4i1.141

Ferone, G. et Lavenka, A. (2015). La classe virtuelle, quels effets sur la pratique de l'enseignant? Distances et médiations des savoirs, 3(10). https://doi.org/10.4000/dms.1047

Feenberg, A. (1989). The written word: on the theory and practice of computer conferencing. Dans R. Mason et A. Kaye (dir.), Mindweave: communication, computers and distance education (p. 22-39). Pergamon.

Garrison, D. R. et Bayaton, M. (1987). Beyond independence in distance education : The concept of control. The American Journal of Distance Education, 1987(3), 3-15.

Ghiglione, R. et Blanchet, A. (1991). Analyse de contenu et contenus d'analyses. Dunod.

Ghiglione, R. et Matalon, B. (1998). Les enquêtes sociologiques. Armand Colin.

Goffman, E. (1973). La mise en scène de la vie quotidienne, Tome 1: La présentation de soi. Les Éditions de minuit.

Harasim, L. (1990). Online Education. Perspective on a New Environnement. Prager.

Henri, F. (1989). La téléconférence assistée par ordinateur dans une activité de formation à distance (Thèse de doctorat, Université de Concordia). https://spectrum.library.concordia.ca/5866/1/NL56075.pdf

Henri, F. (1992). Formation à distance et téléconférence assistée par ordinateur : Interactivité, quasi-interactivité ou monologue ? Revue de l'enseignement à distance, 7(1), 5-24.

Henri, F. et Kaye, A. (1985). Le savoir à domicile, pédagogie et problématique de l'enseignement à distance. Presses de l'Université du Québec - Télé-Université. 
Hilt, R. (1986). The virtual classroom : Using computer-mediated communication for university education. Journal of communication, 1986(36), 95-104.

Jacquinot, G. (1993). Apprivoiser la distance et supprimer l'absence? Ou les défis de la formation à distance. Revue Française de Pédagogie, 1993(102), 55-67.

Jacquinot-Delaunay, G. (2002). Absence et présence dans la médiation pédagogique ou comment faire circuler les signes de la présence. Dans R. Guir, Pratiquer les TICE (p. 103-113). https://doi.org/10.3917/dbu.guir.2002.01.0103

Jorro, A. (2006). L'agir professionnel de l'enseignant. Présenté à Séminaire du centre de Recherche sur la formation, CNAM. https://halshs.archives-ouvertes.fr/halshs-00195900

Kohn, R. C. et Nègre, P. (2003). Les voies de l'observation : Repères pour les pratiques de recherche en sciences humaines. L'Harmattan.

Lamy, T. (1985). La télématique, un outil convivial? Dans F. Henri et A. Kaye, Le savoir à domicile, pédagogie et problématique de l'enseignement à distance (p. 303-328). Presses de l'Université du Québec - Télé-Université.

Le Boterf, G. (2007). Professionnaliser, le modèle de la navigation professionnelle. Éditions d'Organisation.

McBrien, J., Jones, P. et Cheng, R. (2009). Virtual spaces: Employing a synchronous online classroom to facilitate student engagement in online learning. International Review of Research in Open and Distance Learning, 10(3), 1-6.

Macedo-Rouet, M. (2009). La visioconférence dans l'enseignement. Ses usages et effets sur la distance de transaction. Distances et savoirs, 7(1), 65-91.

Marchand, L. (1998). Un changement de paradigme pour un enseignement universitaire moderne. Distances, 2(2), 7-26.

Pastré, P. (2013). Le travail de l'expérience. Dans L. Albarello, J.-M. Barbier, E. Bourgeois et M. Durand, Expérience, activité, apprentissage (p. 93-109). PUF.

Peraya, D. (1995). Les nouvelles technologies ou technologies émergentes: Vers une réappropriation pédagogique des nouvelles technologies? Dans S. Schurch, La formazione a distanza. La formation à distance (p. 17-43). Peter Lang.

Peraya, D. (1999). Médiation et médiatisation : Le campus virtuel. Hermès, 1999(25), 153-168.

Peraya, D. et Dumont, P. (2003). Interagir dans une classe virtuelle: Analyse des interactions verbales médiatisées dans un environnement synchrone. Revue Française de Pédagogie, 2003(145), 51-61.

Poellhuber, B., Racette, N. et Chirchi, M. (2012). De la présence dans la distance par la visioconférence Web. Revue internationale des technologies en pédagogie universitaire / International Journal of Technologies in Higher Education, 9 (1-2), 63-77. https://doi.org/10.7202/1012903ar

Power, T. M. et Desjardins, F. (2012). Mobile learning et classes virtuelles dans les universités canadiennes. Présenté à Les Universités Vivaldi, Caen. http://www.normandie-univ.fr/les-universites-vivaldi-17025.kjsp

Rinaudo, J. L. et Ohana, D. (2007). "Puisqu'ils ont des ordinateurs... ". Discours des enseignants résignés autour du dispositif ordi 35. Présenté à AREF 2007, Strasbourg. http://aref2007.u-strasbg.fr/actes pdf/AREF2007 JeanLuc_RINAUDO_456.pdf

Serres, M. (2003). Interview de mars [Le Journal d'Internet -Marseille].

Thibault, F. (2007). Campus numérique: archéologie d'une initiative ministérielle, Études de communication, Numéro spécial, 17-48.

Verquin Savarieau, B. et Daguet, H. (2012). L'introduction des classes virtuelles synchrones, un moyen de renforcer la qualité de l'accompagnement en formation d'adultes ? frantice.net, 6. http://www.frantice.net/index.php?id=710

Verquin Savarieau, B. et Daguet, H. (2016). La classe virtuelle synchrone une substitution médiatique de l'enseignant pour renforcer la présence en formation à distance ? Sticef, 23(1), 47-75. https://doi.org/10.23709/sticef.23.1.2

Wallet, J. (2007). Le campus numérique FORSE: Analyses et témoignages. Mont-Saint-Aignan: PURH.

Wallet, J. (2012). De la synchronie médiatisée en formation à distance, les classes virtuelles une appellation mal contrôlée. Actes du colloque JOCAIR 2012. Présenté à JOCAIR, Amiens.

Wittorski, R. (2008). La professionnalisation. Savoirs, 17(2), 9-36. https://doi.org/10.3917/savo.017.0009

Zeitler, A. (2013). Construction de l'expérience, habitudes d'interprétation et signification du vécu. Dans L. Albarello, J.-M. Barbier, E. Bourgeois et M. Durand, Expérience, activité, apprentissage (p. 135-162). Paris: PUF. 